\title{
Detection and Doppler monitoring of K2-285 (EPIC 246471491), a system of four transiting planets smaller than Neptune
}


D. Gandolfi ${ }^{3}$, J. Livingston 7 , F. Dai ${ }^{8,9}$, J. C. Morales ${ }^{10,11}$, M. Lafarga ${ }^{10,11}$, S. Albrecht ${ }^{12}$, R. Alonso ${ }^{1,2}$, P. J. Amado ${ }^{13}$, J. A. Caballero ${ }^{14}$, J. Cabrera ${ }^{15}$, W. D. Cochran ${ }^{16}$, Sz. Csizmadiaa ${ }^{15}$, H. Deeg ${ }^{1,2}$, Ph. Eigmüller ${ }^{15,17}$, M. Endl ${ }^{18}$, A. Erikson ${ }^{15}$, A. Fukui ${ }^{19,1}$, E. W. Guenther ${ }^{20}$, S. Grziwa ${ }^{21}$, A. P. Hatzes ${ }^{20}$, J. Korth ${ }^{21}$, M. Kürster ${ }^{22}$, M. Kuzuhara ${ }^{23,24}$, P. Montañes Rodríguez ${ }^{1,2}$, F. Murgas ${ }^{1,2}$, N. Narita ${ }^{23,24,1}$, D. Nespral ${ }^{1,2}$, M. Pätzold $^{21}$, C. M. Persson ${ }^{6}$, A. Quirrenbach ${ }^{25}$, H. Rauer ${ }^{15,16,17}$, S. Redfield ${ }^{26}$, A. Reiners ${ }^{27}$, I. Ribas ${ }^{10,11}$, A. M. S. Smith ${ }^{15}$, V. Van Eylen ${ }^{5}$, J. N. Winn ${ }^{8}$, and M. Zechmeister ${ }^{27}$

(Affiliations can be found after the references)

Received 1 August 2018 / Accepted 12 November 2018

\begin{abstract}
Context. The Kepler extended mission, also known as K2, has provided the community with a wealth of planetary candidates that orbit stars typically much brighter than the targets of the original mission. These planet candidates are suitable for further spectroscopic follow-up and precise mass determinations, leading ultimately to the construction of empirical mass-radius diagrams. Particularly interesting is to constrain the properties of planets that are between Earth and Neptune in size, the most abundant type of planet orbiting Sun-like stars with periods of less than a few years.

Aims. Among many other K2 candidates, we discovered a multi-planetary system around EPIC 246471491, referred to henceforth as K2-285, which contains four planets, ranging in size from twice the size of Earth to nearly the size of Neptune. We aim here at confirming their planetary nature and characterizing the properties of this system.

Methods. We measure the mass of the planets of the K2-285 system by means of precise radial-velocity measurements using the CARMENES spectrograph and the HARPS-N spectrograph.

Results. With our data we are able to determine the mass of the two inner planets of the system with a precision better than $15 \%$, and place upper limits on the masses of the two outer planets.

Conclusions. We find that $\mathrm{K} 2-285 \mathrm{~b}$ has a mass of $M_{\mathrm{b}}=9.68_{-1.37}^{+1.21} M_{\oplus}$ and a radius of $R_{\mathrm{b}}=2.59_{-0.06}^{+0.06} R_{\oplus}$, yielding a mean density of $\rho_{\mathrm{b}}=3.07_{-0.45}^{+0.45} \mathrm{~g} \mathrm{~cm}^{-3}$, while $\mathrm{K} 2-285 \mathrm{c}$ has a mass of $M_{\mathrm{c}}=15.68_{-2.13}^{+2.28} M_{\oplus}$, radius of $R_{\mathrm{c}}=3.53_{-0.08}^{+0.08} R_{\oplus}$, and a mean density of $\rho_{\mathrm{c}}=1.95_{-0.28}^{+0.32} \mathrm{~g} \mathrm{~cm}^{-3}$. For K2-285d $\left(R_{\mathrm{d}}=2.48_{-0.06}^{+0.06} R_{\oplus}\right)$ and $\mathrm{K} 2-285 \mathrm{e}\left(R_{\mathrm{e}}=1.95_{-0.05}^{+0.05} R_{\oplus}\right)$, the upper limits for the masses are $6.5 M_{\oplus}$ and $10.7 M_{\oplus}$, respectively. The system is thus composed of an (almost) Neptune-twin planet (in mass and radius), two sub-Neptunes with very different densities and presumably bulk composition, and a fourth planet in the outermost orbit that resides right in the middle of the super-Earth/sub-Neptune radius gap. Future comparative planetology studies of this system would provide useful insights into planetary formation, and also a good test of atmospheric escape and evolution theories.
\end{abstract}

Key words. planetary systems - planets and satellites: dynamical evolution and stability - planets and satellites: detection planets and satellites: fundamental parameters - planets and satellites: atmospheres

\section{Introduction}

Space-based transit surveys such as CoRoT (Auvergne et al. 2009) and Kepler (Borucki et al. 2010) have revolutionized the field of exoplanetary science. Their high precision and nearly uninterrupted photometry has opened the doors to explore planet parameter spaces that are not easily accessible from the ground, most notably the Earth-radius planet domain. However, our knowledge of both super-Earths $\left(R_{p}=1-2 R_{\oplus}\right.$ and $M_{p}=1-$ $\left.10 M_{\oplus}\right)$ and Neptune planets $\left(R_{p}=2-6 R_{\oplus}\right.$ and $\left.R_{p}=10-40 M_{\oplus}\right)$ is still limited, due to the small radial velocity (RV) variation induced by such planets and the relative faintness of most Kepler host stars $(V>13 \mathrm{mag})$ which make precise mass determinations difficult.

Many questions therefore remain unanswered, such as for example those referring to the composition and internal structure of small planets. Fulton et al. (2017) and Fulton \& Petigura (2018) reported a radius gap at $\sim 2 R_{\oplus}$ in the exoplanet radius distribution using Kepler data for Sun-like stars, and Hirano et al. (2018) indicated that the gap could extend down to the $\mathrm{M}$ dwarf domain. This would point to a very different planetary nature for planets on each side of the gap. Is this due to planet migration? Are the larger planets surrounded by a H/He atmospheres while the smaller planet have lost these envelopes? Or, did they already form with very different bulk densities? Answering these questions requires statistically significant samples of well-characterized small planets, especially in terms of orbital parameters, mass, radius and mean density.

The extended K2 mission of Kepler is a unique opportunity to gain knowledge about small close-in planets. Every 3 months, K2 observes a different stellar field located along the ecliptic, targeting stars that are up to 15 times brighter than those targeted by the original Kepler mission. The KESPRINT collaboration ${ }^{1}$ is an international effort dedicated to the discovery, confirmation,

http://www.iac.es/proyecto/kesprint 
and characterization of planet candidates from the space transit missions K2 and TESS and, in the future, PLATO. We have been focusing on determining the masses of small planets around bright stars, especially for planets in or around the radius gap.

Here, we present the discovery and characterization of four transiting planets around the star EPIC 246471491 (K2-285). While these planets are observed to have radii between 2 and 3.5 times greater than that of the Earth, our follow-up observations indicate that they have very different bulk compositions. This has significant implications for the physical nature of planets around the radius gap. In this paper we provide ground-based follow-up observations that confirm that EPIC 246471491 is a single object and establish its main stellar properties. We also jointly analyze the K2 data together with high-precision RV data from CARMENES and HARPS-N spectrographs, to retrieve orbital solutions and planetary masses. Finally, we discuss the possible bulk compositions of the planets, leading to different densities.

\section{K2 photometry and candidate detection}

EPIC 246471491 $(\mathrm{RA}=23: 17: 32.23, \mathrm{Dec}=01: 18: 01.04$, in the Aquarius constellation) was proposed as a K2 GO target for Campaign 12 in several programs (GO-12123, PI Stello; GO-12049, PI Quintana; and GO-12071, PI Charbonneau). The star was observed for 78.85 days from 15 December 2016 to 4 March 2017. During this interval, the Kepler spacecraft entered safe mode from 1 to 6 February 2017, causing a gap of 5.3 days in the data.

We built the light curve of EPIC 246471491 directly from raw data (files downloaded from the Mikulski Archive for Space Telescopes ${ }^{2}$, MAST), using the long cadence (LC) version (29.4 min time stamps). Our pipeline is based on the implementation of the pixel level decorrelation (PLD) model (Deming et al. 2015), and a modified version of the Everest ${ }^{3}$ pipeline (Luger et al. 2018). The PLD model uses a Taylor expansion of the instrumental signal as regressors in a linear model. These regressors are the products of the fractional fluxes in each pixel of the target aperture. The optimal aperture is built by searching for the photo-center and selecting pixels with a threshold of $1.2 \sigma$ over the previously calculated background (Fig. 1). The pipeline extracts the raw light curve from the apertures, removing time cadences with bad quality flags, and the background contribution. Next, it fits a regularized regression model to the data, iteratively up to the third order, and applies the cross-validation method to obtain the regularization matrix coefficients and Gaussian processes to compute the covariance matrix. All these steps are described in Luger et al. (2018).

Prior to planet searches, we need to flatten the K2 light curve by iteratively applying a robust locally weighted regression method (Cleveland 1979) until no outliers are detected. We remove $3 \sigma$ outliers replacing these points by the median of the neighbors. We note that the first two days and the last day of data, which show anomalies probably related to thermal settling, were removed from our analysis. Applying these methods iteratively, we are able to remove any stellar flares. We then divide the original light curve by this variability model. The initial and final detrended K2 light curves are plotted in Fig. 2.

Subsequently, we present a Box-fitting Least Squares (BLS) algorithm (Kovács et al. 2002) used to detect the period of each

\footnotetext{
2 https://archive.stsci.edu/kepler/data_search/search. php

3 https://github.com/rodluger/everest
}

EPIC 246471491


Fig. 1. K2 image of the object EPIC 246471491. A custom-built changing aperture is fitted based on the pixel counts of the star and background. The image shows three typical apertures used at the beginning (top left panel), middle (top right panel) and end (bottom left panel) of the time series. Bottom right panel: a high-resolution image of the same field taken from Palomar Observatory.

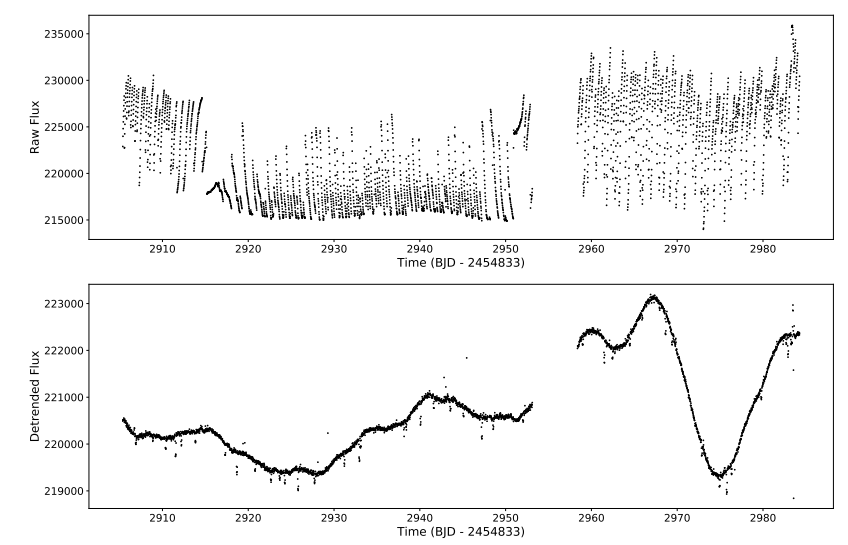

Fig. 2. Kepler light curves of EPIC 246471491. Top panel: original raw light curves as derived from raw flux data. Bottom panel: detrended light curve after analysis with our modified Everest-based pipeline. Stellar variability of the order of tens of days and the transits of several planets are clearly visible.

possible planetary signal in the light curve. The BLS algorithm is very sensitive to outliers, and therefore we remove them by performing a sigma clipping. In this case, a value of $30 \sigma$ is enough. Once a planetary signal is detected in the power spectrum, the BLS provides the epoch and the period of the candidate. We then remove this signal from the light curve by masking all points in transit, that is, we mark the times of the $T_{0}$ epoch and the $T_{0}$ plus $N$ times the period, and mask all points that fall in a window centered on these times with a width equal to the transit duration. In this manner, we iteratively remove every single transit until no signal is found by BLS.

Four planet detections were made in the course of the analysis of EPIC 246471491, at periods of 3.47, 7.13, 10.45, and 14.76 days (see Fig. 3). The planet periods are close to $1: 2: 3: 4$ commensurability, but not quite, the precise ratio numbers being $1: 2.05: 3.01: 4.25$. This near-commensurability 



Fig. 3. Phase-folded transit light curves of the four planets detected orbiting EPIC 246471491. Top panels: transit light curves and best-fit transit models (red) on the same flux scale. Lower panels: residuals of the transit fit. may be indicative that the system is in resonance. Figure 3 shows the phase-folded light curve for each transit, and its bestfit transit model. We fit the phase-folded transit light curve of each planet with the python package batman (Kreidberg 2015). We tentatively fit the different planets with a nonlinear least-squares minimization routine, which yielded good results for the transit parameters, and took these as input for the Markov chain Monte Carlo (MCMC) algorithm implemented in emcee (Foreman-Mackey et al. 2013), which was used to sample from the joint posterior distribution, using 100 walkers and 30000 steps. We then removed the first 22500 steps to estimate the uncertainties in the transit parameters. Once we obtain the MCMC results for the transit parameters of a planet, we iteratively remove the points inside the transit for the next fitting. The retrieved planetary parameters derived from fitting the $\mathrm{K} 2$ light curve alone are given in Table A.1.

The auto-correlation analysis of the $\mathrm{K} 2$ photometry retrieves a stellar rotational period at around 15 days, but the autocorrelation peak is broad and insignificant. We discuss this point further in Sect. 5.

\section{Ground-based follow-up observations}

\subsection{Lucky imaging and $A O$ observations}

We performed Lucky Imaging (LI) of EPIC 246471491 with the FastCam camera (Oscoz et al. 2008) at the 1.52-m Telescopio Carlos Sánchez (TCS), and with the NASA Exoplanet Star and Speckle Imager (NESSI; Scott et al. 2016, 2018) on the 3.5-m WIYN telescope at the Kitt Peak National Observatory. Both results strongly constrained the possibility that the observed transit signals come from a nearby faint star. However, these upper limits (not shown here) were superseded by adaptive optics imaging at SUBARU.

We obtained high-resolution images for EPIC 246471491 using the InfraRed Camera and Spectrograph (IRCS; Kobayashi et al. 2000) and the adaptive-optics (AO) system on the Subaru 8.2-m telescope on UT 2018 June 14. To check for the absence of nearby companions, we imaged the target in the $K^{\prime}$ band with the fine-sampling mode ( 1 pix $=20$ mas $)$, and implemented two types of sequences with a five-point dithering. For the first sequence we used a neutral-density (ND) filter, the transmittance of which is $\sim 0.81 \%$ in the $K^{\prime}$ band to obtain unsaturated frames for the absolute flux calibration. We then acquired saturated frames to look for faint nearby companions. The total integration times amounted to 450 and $45 \mathrm{~s}$ for the unsaturated and saturated frames, respectively. We reduced and median-combined those frames following the procedure described in Hirano et al. (2016). The combined images revealed no nearby companion around EPIC 246471491. To check for the detection limit, we drew the $5 \sigma$ contrast curve following Hirano et al. (2016) based on the combined saturated image. As plotted in Fig. $4, \Delta m_{K^{\prime}}=7$ was achieved at $\sim 0$.' 5 from the target. The inset of the figure displays the target image with field-of-view of $4^{\prime \prime} \times 4^{\prime \prime}$.

\subsection{CARMENES radial velocity observations}

Radial-velocity measurements of EPIC 246471491 were taken with the CARMENES spectrograph, mounted at the $3.5-\mathrm{m}$ telescope at the Calar Alto Observatory in Spain. The CARMENES instrument has two arms (Quirrenbach et al. 2014), the visible (VIS) arm covering the spectral range $0.52-0.96 \mu \mathrm{m}$ and a near-infrared (NIR) arm covering the $0.96-1.71 \mu \mathrm{m}$ range. Here we use only the VIS channel observations to derive 




Fig. 4. Subaru AO image (inset) and $5 \sigma$ contrast light curve of EPIC 246471491. The inset image has a FOV of $4^{\prime \prime} \times 4^{\prime \prime}$.

RV measurements. The overall instrumental performance of CARMENES has been described by Reiners et al. (2018).

A total of 29 measurements were taken over the period 2017 September 20-2017 December 17, covering a time span of 98 days. In all cases exposure times were set at 1800 s. Radialvelocity values, chromatic index (CRX), differential linewidth (dLW), and $\mathrm{H} \alpha$ index were obtained using the SERVAL program (Zechmeister et al. 2018). For each spectrum, we also computed the cross-correlation function (CCF) and its full width half maximum (FWHM), contrast (CTR), and bisector velocity span (BVS) following Reiners et al. (2018). The RV measurements are given in Table 1, corrected for barycentric motion, secular acceleration and nightly zero-points. For more details, see Trifonov et al. (2018) and Luque et al. (2018).

\subsection{HARPS-N RV observations}

Radial-velocity measurements were also taken with the HARPS-North spectrograph, mounted at the 3.5-m TNG telescope at the Roque de los Muchachos Observatory in Spain. The HARPS-N instrument (Cosentino et al. 2012) covers the spectral range $0.383-0.693 \mu \mathrm{m}$. In total, nine HARPS-N measurements were taken over the period 16 September 2017 to 10 January 2018, covering a time span of 112 days. Exposure times were set at $3600 \mathrm{~s}$. To derive radial velocities, SERVAL was also applied to the data. The performance of SERVAL RV extraction compared to the standard HARPS and HIRES pipelines is described in Trifonov et al. (2018). Both CARMENES and HARPS-N RV measurements are given in Table 1.

\section{Host star properties}

To retrieve the physical properties of EPIC 246471491, we analyzed the co-added, RV-corrected CARMENES spectra using the Spectroscopy Made Easy (SME) code (Piskunov \& Valenti 2017) which is designed to derive the fundamental parameters of stars. It iteratively calculates the synthesized spectrum based on a large grid of model spectra. The synthesized spectrum is fitted to the observed spectra using a $\chi^{2}$ minimization process. In this case, we used one-dimensional MARCS models (Gustafsson et al. 2008). Providing the code with fixed turbulent velocities $v_{\mathrm{mac}}=2.5 \pm 0.7 \mathrm{~km} \mathrm{~s}^{-1}$ (Doyle et al. 2014) and $v_{\text {mic }}=0.82 \pm 0.4 \mathrm{~km} \mathrm{~s}^{-1}$ (Bruntt et al. 2010), we solved for $T_{\text {eff }}$ by analyzing the Balmer profile of $\mathrm{H} \alpha, \log g_{\star}$ by fitting the Ca I triplet at 6102, 6122 and $6162 \AA$, and $[\mathrm{Fe} / \mathrm{H}]$ and $v \sin i$ by
Table 1. Radial-velocity measurements derived from HARPS-N and CARMENES observations used in this paper.

\begin{tabular}{lccc}
\hline \hline JD & RV $\left(\mathrm{m} \mathrm{s}^{-1}\right)$ & Error $\left(\mathrm{m} \mathrm{s}^{-1}\right)$ & Instrument \\
\hline 2458013.4565 & 2.72 & 1.06 & HARPS-N \\
2458013.59056 & -0.57 & 1.01 & HARPS-N \\
2458014.47738 & -0.13 & 1.44 & HARPS-N \\
2458014.61324 & -2.95 & 1.42 & HARPS-N \\
2458015.49584 & 0.68 & 1.92 & HARPS-N \\
2458015.53839 & -3.43 & 1.38 & HARPS-N \\
2458017.35179 & 12.56 & 4.67 & CARMENES \\
2458017.49193 & 8.79 & 3.27 & CARMENES \\
2458019.58022 & 14.81 & 3.26 & CARMENES \\
2458020.56477 & 20.69 & 3.24 & CARMENES \\
2458021.44042 & 0.44 & 5.90 & CARMENES \\
2458021.64733 & 5.40 & 4.63 & CARMENES \\
2458022.3294 & -1.55 & 4.09 & CARMENES \\
2458022.63247 & -3.02 & 4.87 & CARMENES \\
2458023.35717 & -2.41 & 3.49 & CARMENES \\
2458023.46814 & -4.80 & 3.69 & CARMENES \\
2458024.57625 & -13.15 & 4.36 & CARMENES \\
2458026.42467 & -9.19 & 3.09 & CARMENES \\
2458046.47123 & -6.38 & 1.32 & HARPS-N \\
2458047.43121 & -5.17 & 2.57 & CARMENES \\
2458048.35116 & -0.46 & 3.01 & CARMENES \\
2458049.37702 & -4.61 & 2.72 & CARMENES \\
2458050.33568 & -9.29 & 3.02 & CARMENES \\
2458050.43032 & -8.37 & 3.80 & CARMENES \\
2458051.30778 & -2.47 & 2.62 & CARMENES \\
2458051.49481 & -0.60 & 2.60 & CARMENES \\
2458052.34674 & -1.01 & 2.48 & CARMENES \\
2458052.58013 & -14.27 & 6.17 & CARMENES \\
2458053.4422 & -2.72 & 3.09 & CARMENES \\
2458053.54225 & 1.46 & 4.47 & CARMENES \\
2458054.37099 & 8.08 & 3.74 & CARMENES \\
2458054.45669 & 0.80 & 3.16 & CARMENES \\
2458080.36219 & 13.81 & 1.27 & HARPS-N \\
2458099.31923 & 6.61 & 8.59 & CARMENES \\
2458104.27631 & 11.03 & 7.81 & CARMENES \\
2458105.29813 & 14.83 & 6.32 & CARMENES \\
2458129.3238 & 12.10 & 1.75 & HARPS-N \\
\hline & & &
\end{tabular}

fitting approximately $50 \mathrm{Fe}$ lines. We find $T_{\text {eff }}=4975 \pm 95 \mathrm{~K}$, $\log g_{\star}=4.4 \pm 0.1 \mathrm{dex},[\mathrm{Fe} / \mathrm{H}]=0.00 \pm 0.05 \mathrm{dex}$, and $v \sin i=$ $3.9 \pm 0.8 \mathrm{~km} \mathrm{~s}^{-1}$, respectively. See Table 2 for a summary of EPIC 246471491 stellar parameters.

We confirmed the effective temperature and the value for $\log g_{\star}$ by also modeling the Na I doublet $(5889.95 / 5895.9 \AA)$ using SME and deriving the abundance for $\mathrm{Na}$ I from fainter lines in our CARMENES spectrum. Also, by analyzing the equivalent width of the interstellar sodium components (Poznanski et al. 2012), we find an extinction of $E(B-$ $V)=0.02 \pm 0.003$ that corresponds to $A_{V}=0.07 \pm 0.01 \mathrm{mag}$.

We also used the HARPS-N co-added spectrum to derive stellar parameters. In particular, we fitted the spectral energy distribution using low-resolution model spectra with the same spectroscopic parameters as those found using the CARMENES co-added spectrum. Our results return an interstellar reddening value of $A_{V}=0.1 \pm 0.05 \mathrm{mag}$.

We then used the $T_{\text {eff }}$ and $[\mathrm{Fe} / \mathrm{H}]$ values retrieved by SME, along with the new Gaia parallax value of $\pi=6.43 \pm 0.11$ mas (Lindegren et al. 2018). We quadratically added 0.1 mas to the 
Table 2. Stellar parameters of EPIC 246471491 (K2-285).

\begin{tabular}{lr}
\hline \hline \multicolumn{2}{c}{ EPIC 246471491 } \\
\hline RA $^{a}(\mathrm{~J} 2000.0)$ & $23: 17: 32.23$ \\
$\operatorname{Dec}^{a}(\mathrm{~J} 2000.0)$ & $01: 18: 01.04$ \\
$V$-band magnitude $^{b}(\mathrm{mag})$ & $12.030 \pm 0.121$ \\
Spectral type $^{b}$ & $\mathrm{~K} 2 \mathrm{~V}$ \\
Effective temperature $^{b} T_{\text {eff }}(\mathrm{K})$ & $4975 \pm 95$ \\
Surface gravity $^{b} \log g_{\star}(\mathrm{cgs})$ & $4.4 \pm 0.1$ \\
Iron abundance $^{b}[\mathrm{Fe} / \mathrm{H}](\mathrm{dex})$ & $0.0 \pm 0.05$ \\
Mass $^{b} M_{\star}\left(M_{\odot}\right)$ & $0.830 \pm 0.023$ \\
Radius $^{b} R_{\star}\left(R_{\odot}\right)$ & $0.787 \pm 0.016$ \\
Projected rot. velocity $^{b} v \sin i\left(\mathrm{~km} \mathrm{~s}^{-1}\right)$ & $3.9 \pm 0.8$ \\
Microturbulent velocity $^{c} v_{\text {mic }}\left(\mathrm{km} \mathrm{s}^{-1}\right)$ & $0.82($ fixed $)$ \\
Macroturbulent velocity $^{d} v_{\text {mac }}\left(\mathrm{km} \mathrm{s}^{-1}\right)$ & 2.5 (fixed) \\
Interstellar reddening $^{b} A_{\mathrm{v}}(\mathrm{mag})$ & $0.07 \pm 0.01$ \\
Distance $^{e}(\mathrm{pc})$ & $155.6 \pm 6.4$ \\
\hline
\end{tabular}

Note. ${ }^{(a)}$ Hipparcos, the New Reduction (van Leeuwen 2007). ${ }^{(b)}$ This work and AAVSO (https : //www . aavso. org/). ${ }^{\left({ }^{(}\right)}$Bruntt et al. (2010). ${ }^{(d)}$ Doyle et al. (2014). ${ }^{(e)}$ Gaia DR2 (Lindegren et al. 2018).

nominal uncertainty of Gaia to account for systematics (see Luri et al. 2018).

The stellar magnitude in $V$ band is taken from the AAVSO Photometric All Sky Survey (APASS) and corrected for extinction. The PARAM ${ }^{4}$ models (da Silva et al. 2006) return a stellar mass of $M_{\star}=0.830 \pm 0.023 M_{\odot}$, a radius of $R_{\star}=0.787 \pm 0.016 R_{\odot}$ and a $\log g_{\star}=4.539 \pm 0.024 \mathrm{cgs}$. The latter value of surface gravity is consistent with the SME value within less than $2 \sigma$. As a sanity check, we used the bolometric correction from Torres et al. (2010) and got a radius of $R_{\star}=0.880 \pm 0.080 R_{\odot}$, which is roughly consistent with the previous value.

\section{Frequency analysis of relative velocity and photometric data}

We performed a frequency analysis of the available RV observations. In Fig. 5, we plot the generalized LombScargle periodogram (GLS; Zechmeister \& Kürster 2009) of the CARMENES RV data. For each periodogram, we compute the theoretical false-alarm probability (FAP) following Zechmeister \& Kürster (2009), and mark the 10, 1, and $0.1 \%$ significance levels. The vertical red lines mark the orbital frequencies of planets b, c, d and e, and the thick blue lines mark the stellar rotational frequencies associated with stellar variability.

It is easily seen that the dominant signals in the CARMENES periodogram are those at 12.1 days and $\sim 22$ days. These periodicities are suggested in the CARMENES chromatic index (CRX), but with FAP $>10 \%$, an indicator developed for CARMENES data to recognize wavelength-dependent variability attributable to stellar activity (Zechmeister et al. 2018), the dLW, the CCF BVS, and the CCF FWHM indices. Based solely on the data available to us, it is not clear which one of the two periods is the true rotational period of the star, and which one is indeed an alias or a harmonic of the other.

There is evidence that $\sin i$ should be in fact close to unity for these types of systems (Winn et al. 2017). A simple calculation $\left(P_{\text {rot }} \sin i=2 \pi R v \sin i\right)$, using the stellar $v \sin i$

\footnotetext{
4 http://stev.oapd.inaf.it/
}

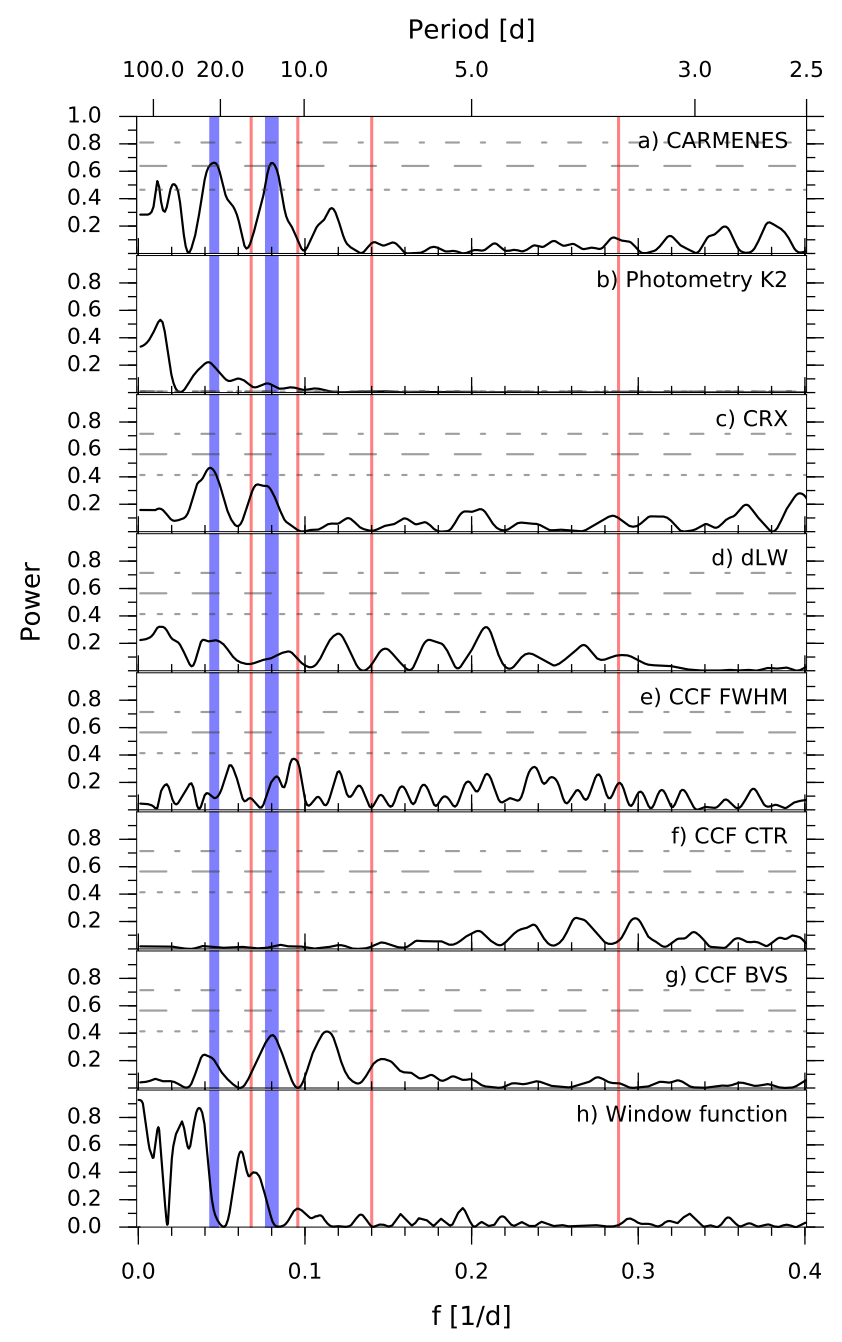

Fig. 5. Generalized Lomb-Scargle periodograms (GLS) of the EPIC $246471491 \mathrm{RV}$ s from CARMENES data (panel $a$ ), the K2 photometry (panel $b$ ), and the CRX (panel $c$ ), dLW (panel $d$ ), FWHM (panel e), CTR (panel f), and BVS (panel $g$ ) indices. Panel $h$ : window function of the data. Vertical red lines indicate the frequencies associated with each of the four transiting planets, and the blue vertical lines mark the frequencies associated with the activity of the host star. The highest peaks in the CARMENES GLS are located at $f=0.045 \mathrm{~d}^{-1}$ $(P \sim 22 \mathrm{~d})$ and $f=0.081 \mathrm{~d}^{-1}(P=12.1 \mathrm{~d})$ and are linked to the rotation of the star. These periodicities are also significant in the K2 photometry and the CRX index. Horizontal lines show the FAP levels of $10 \%$ (short-dashed line), $1 \%$ (long-dashed line) and $0.1 \%$ (dot-dashed line).

value and assuming $\sin i=1$ gives an expected stellar rotational period upper limit of $10.2_{-1.7}^{+2.6}$ days. Therefore, we adopt 12.1 days as the true rotational period of the star, $P_{\text {rot }}$, seen in the CARMENES GLS periodogram. The $\sim 22$ days peak is then an alias originated from the window function peak at $\sim 27.5$ days, caused by our scheduling of optimal observations along the lunar cycle.

In light of these results, it is clear that the dominant signal in the RV data is that of stellar activity, and that this needs to be taken into account in order to retrieve the planetary masses. In Fig. 6 we show the GLS periodograms of the CARMENES data with the stellar and planetary periodicities marked, the spectral power being dominated by the former. In the middle panel, we filter the data by removing the $P_{\text {rot }}$ periodicity. We do this by fitting the amplitude and phase of a sinusoidal signal, and 




Fig. 6. Generalized Lomb-Scargle periodograms (GLS) of the EPIC 246471491 RVs from CARMENES and HARPS-N. Panel $a$ : same data and analysis is shown as panel a of Fig. 5. Panel $b$ : GLS of the CARMENES data after removing the prominent peak at $f=0.081 \mathrm{~d}^{-1}$, corresponding to $P_{\text {rot }}=12.1$ days. In this case the frequency associated with the alias peak at $\sim 22$ days also loses all the power, and the higher peaks are located at the periodicities of the four planets. Panel $c$ : HARPS-N data (also $P_{\text {rot }}$ corrected) are added to the CARMENES data. The joint GLS shows significant periodicities for two of the four planets in this system. As in Fig. 5, horizontal lines show the FAP levels of 10 (short-dashed line), 1 (long-dashed line), and $0.1 \%$ (dot-dashed line).

computing the GLS periodogram of the residuals of this fit, in the same way as is done for planetary signals.

This procedure eliminates both the 12.1-day and 22-day signals, confirming that the latter is in fact an alias. Now the major peaks in the power spectra correspond to the planetary orbital periods, although they are not above the FAP $=10 \%$ level. In the bottom panel, the HARPS-N data are added accounting for the RV offset between both instruments using the measurements taken in consecutive days with HARPS-N (JD 2458046.5) and CARMENES (JD 2458047.5). Removing $P_{\text {rot }}$ signal from HARPS-N data does not have a strong effect on the final result. Regardless, for the sake of consistency, we removed it in our analysis. A possible explanation may lie in the fact that there is only a handful of measurements (9), or that the HARPS-N and CARMENES spectrographs cover different spectral ranges and therefore their sensitivity to stellar activity is different. The GLS periodogram of the combined data shows significance peaks

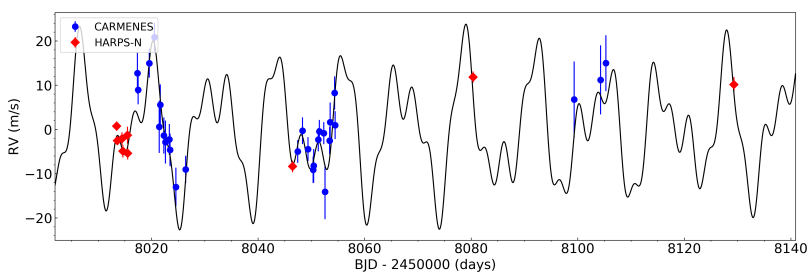

Fig. 7. Time series of the RV measurements of K2-285 derived from CARMENES (blue dots) and HARPS-N (red diamonds). The black line corresponds to the best-fit model to the data, which includes the RV signal of each of the four planets and the stellar activity.

(FAP $\approx 0.1 \%$ ) at the orbital periods of planets $\mathrm{c}$ and $\mathrm{d}$, and above $\mathrm{FAP}=10 \%$ for planets $\mathrm{b}$ and $\mathrm{e}$.

\section{Joint analysis and mass determinations}

In order to retrieve the masses of the planets in the EPIC 246471491 system, we performed a joint analysis of the photometric $\mathrm{K} 2$ data and the RV measurements from CARMENES and HARPS-N. We make use of the Pyaneti ${ }^{5}$ code (Barragán et al. 2016), which uses MCMC techniques to infer posterior distributions for the fitted parameters. The RV data are fitted with Keplerian orbits, The transit model follows the quadratic limb-darkened law of Mandel \& Agol (2002). For the linear $\mathrm{u} 1$ and quadratic $\mathrm{u} 2$ limb-darkening coefficients, we use the $q 1=(u 1+u 2)^{2}$ and $q 2=u 1(2(u 1+u 2))^{-1}$ parameterization described in Kipping (2013). For $q 1$ and $q 2$ we set uninformative uniform priors in the range $[0,1]$ to sample a physical solution for the limb-darkening coefficients. These methods have already been successfully applied to several planets; see for example Niraula et al. (2017) or Prieto-Arranz et al. (2018) for details.

Although no coherent rotational modulation is found in the K2 data alone, as in Prieto-Arranz et al. (2018), the light curve of EPIC 246471491 suggests that the evolution timescale of active regions is longer than the K2 observations (80 days). Since our combined observations cover 112 days, we decided to model the stellar activity signal with a sinusoid (Pepe et al. 2013; Barragán et al. 2018). Therefore, on top of the planetary signals, we include in the fit a fifth RV signal corresponding to the stellar variability at $P_{\text {rot }}$, which we identified as the dominant RV signal in the previous section. Van Eylen \& Albrecht (2015) reported that the eccentricity of small planets in Kepler multi-planet systems is low. Given that K2-285 is a compact short-period multiplanetary system, we also assumed tidal circularization of the orbits and fixed the eccentricity to zero for all four planets.

Figure 7 shows the combined CARMENES and HARPS-N RV measurements plotted against time, with a superimposed best-fit model containing the RV variations due to the four planets and a stellar activity signal. In our analysis, we did not discard RV observations that were obtained during transits, but the expected Rossiter-McLaughlin amplitude is negligible.

The individual phased RV signals for each of the four planets, once the stellar variability signal and the signals from the other planets have been removed, are shown in Fig. 8. Also shown is the phased stellar activity signal once the signals of the four planets have been removed. The residuals around the best-fit model are shown below each panel. The RV signal of the stellar activity is readily detectable and has the largest RV semi-amplitude. We can also identify at larger than $3 \sigma$ significance level the semiamplitudes of planets $b$ and $c$, while we can only place upper

https://github.com/oscaribv/pyaneti 

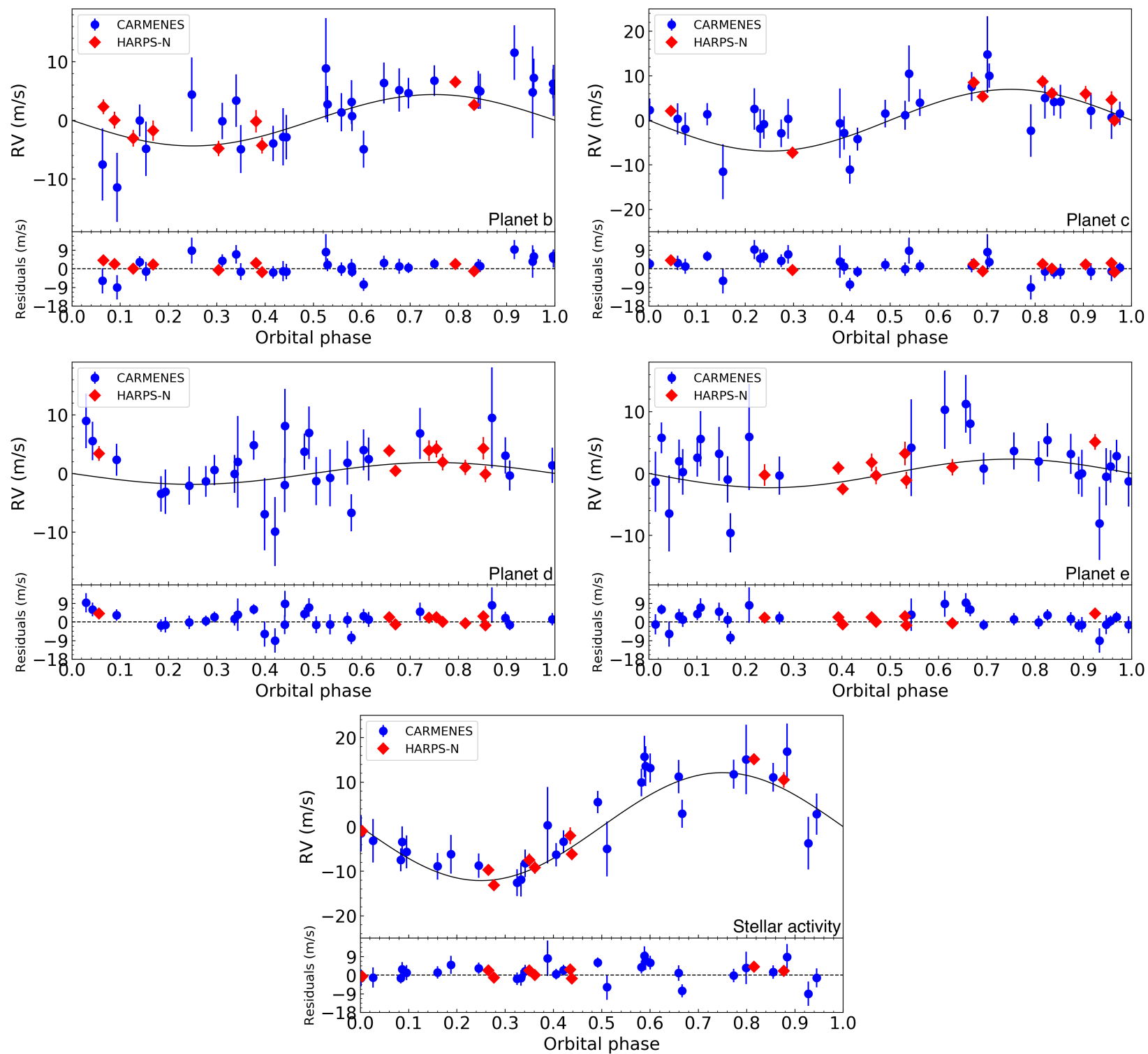

Fig. 8. Top left panel: phase-folded RV measurements of K2-285 over the period of planet b, after eliminating the signal from planets c, d, and e, and the stellar activity signal. Top right panel: as in the top-left panel but for planet c. Middle left panel: as in the top-left panel but for planet d. Middle right panel: as in the top-left panel but for planet e. Bottom panel: as in the top-left panel but for the stellar activity signal. The derived semiamplitudes for these planets are $4.62_{-0.65}^{+0.58} \mathrm{~m} \mathrm{~s}^{-1}, 5.90_{-0.80}^{+0.86} \mathrm{~m} \mathrm{~s}^{-1}, 0.68_{-0.50}^{+0.77} \mathrm{~m} \mathrm{~s}^{-1}$ and $1.69_{-0.72}^{+0.74} \mathrm{~m} \mathrm{~s}^{-1}$, while the stellar activity signal has an amplitude of $12.29_{-0.81}^{+0.80} \mathrm{~m} \mathrm{~s}^{-1}$. This translates into a mass determination of $9.68_{-1.37}^{+1.21} M_{\oplus}$ and $15.68_{-2.13}^{+2.28} M_{\oplus}$ for planets b and c, respectively, and upper-limit mass determinations of $6.5 M_{\oplus}$ and $10.7 M_{\oplus}$ (at $99 \%$ confidence level) for planets d and e, respectively.

limits on the masses of planets d and e. In Table A.1 the planet properties of the K2-285 system are summarized.

As a further test, we used the code SOAP2 (Dumusque et al. 2014) to estimate the expected induced RV signal coming from stellar activity. We assume that spots generate a flux decrement of $1.5 \%$ from the largest depth in the light curve (Fig. 2). We used the stellar parameters from Table 2 and a stellar rotation period of 12.3 days. We assume that the star has two spots separated by $180^{\circ}$ located at the stellar equator. The output of SOAP2 gives an expected induced RV signal of $\sim 13 \mathrm{~m} \mathrm{~s}^{-1}$. This result is consistent with the fitted amplitude in our model.

\section{Discussion and conclusions}

We determined masses, radii, and densities for two of the four planets known to transit $\mathrm{K} 2-285$. We find that $\mathrm{K} 2-285 \mathrm{~b}$ has a mass of $M_{\mathrm{b}}=9.68_{-1.37}^{+1.21} M_{\oplus}$ and a radius of $R_{\mathrm{b}}=2.59_{-0.06}^{+0.06} R_{\oplus}$, yielding a mean density of $\rho_{\mathrm{b}}=3.07_{-0.45}^{+0.45} \mathrm{~g} \mathrm{~cm}^{-3}$, while K2-285c has a mass of $M_{\mathrm{c}}=15.68_{-2.13}^{+2.28} M_{\oplus}$, radius of $R_{\mathrm{c}}=3.53_{-0.08}^{+0.08} R_{\oplus}$, and a mean density of $\rho_{\mathrm{c}}=1.95_{-0.28}^{+0.32} \mathrm{~g} \mathrm{~cm}^{-3}$. For K2-285d and $\mathrm{K} 2-285 \mathrm{e}$, we are able to calculate upper limits for the masses at $6.5 M_{\oplus}$ and $10.7 M_{\oplus}$, respectively. The upper limits are determined by multiplying the semi-amplitude upper limit of the error bar by three (3-sigma error) and adding this value to that of the value of the semi-amplitude. The resulting semi-amplitude value is then transformed into an upper mass limit.

Fulton et al. (2017) and Van Eylen et al. (2018) reported a bi-modal distribution in the radii of small planets at the boundary between super-Earths and sub-Neptunes. A clear distinction between two different families of planets is reported: on the one hand, super-Earths have a radius distribution that peaks at $R_{\mathrm{p}} \sim 1.5 R_{\oplus}$, and on the other sub-Neptune planets have a radius 




Fig. 9. Mass-radius diagram for all known planets with masses in the range $0.5-20 M_{\oplus}$ and radius $1-4 R_{\oplus}$, comprising Earth-like to superEarth to sub-Neptune regimes. Data are taken from the TEPCat database (Southworth 2011). Planets belonging to multiple systems are marked as open gray dots while single planets are marked as solid gray dots. The four planets of the K2-285 system are marked in different colors. Theoretical models for the internal composition of the planets are taken from Zeng et al. (2016).

distribution that peaks at $R_{\mathrm{p}} \sim 2.5 R_{\oplus}$. These two populations are separated by a gap in the radius distribution.

Figure 9 illustrates the mass-radius diagram of all known planets with precise mass determination, extending the full parameter space encompassing Earth-like, super-Earth, and Neptune planets $\left(1-4 R_{\oplus}, 0.5-20 M_{\oplus}\right)$. The four planets of the K2-285 system are also plotted. Two of the planets, $b$ and d, fall in the sub-Neptune category, with radius very close to one of the peaks of the bi-modal distribution at $2.5 R_{\oplus}$, while planet e belongs to the scarce population of planets located within the radius gap. Planet $\mathrm{c}$ is a larger object with only a slightly smaller radius and larger density than Neptune (3.9 vs. $3.5 R_{\oplus}$ and 1.64 vs. $1.95 \mathrm{~g} \mathrm{~cm}^{-3}$; for Neptune and K2-285c, respectively).

Using the values in Table A.1, the estimated transmission signals corresponding to $\mathrm{H} / \mathrm{He}$ atmospheres (which would be the optimistic case for super-Earth size planets) of the four planets would be of 20,32, 21, and 8 ppm for planets b, c, d, and e, respectively. For planets $d$ and $e$, the upper mass limit has been used for the calculations, so presumably the true signals would be larger. Still, with such relatively small atmospheric signatures, the planets are not optimal for transmission spectroscopy studies using current instrumentation due to the faintness of the parent star.

However, as in the case of the triple transiting system K2-135 (Niraula et al. 2017; Prieto-Arranz et al. 2018), the four planets around K2-285 could provide a great test case to study comparative atmospheric escape and evolution within the same planetary system. From Fig. 9 it is readily seen that the two planets with well-determined mass have very different densities. Planet b has a bulk density close to pure water, while planet $\mathrm{c}$ is a much more inflated, lower-density planet. Assuming that all planets in the system were formed with similar composition, the different bulk densities could be explained by the factor-five larger insolation flux received by planet $\mathrm{b}$ compared to $\mathrm{c}$, driving atmospheric escape and mass loss. While the masses of the other two planets are only upper limits, planet $\mathrm{d}$ (the third in distance from the star) clearly falls in the low-density regime, which would be consistent with this hypothesis. For planet e, a larger range in densities is possible, from pure $\mathrm{MgSiO}_{3}$ to extremely low densities. Therefore, to further research this possibility, precise measurements of the ultraviolet flux of K2-285 and comparative atmospheric studies using high-resolution transmission spectroscopy focused on exosphere and atmospheric escape processes through the detection of $\mathrm{H} \alpha$, $\mathrm{Ly} \alpha$, or He lines should be conducted in the near future with the next generation of Extremely Large Telescopes (ELTs).

Acknowledgements. CARMENES is an instrument for the Centro Astronómico Hispano-Alemán de Calar Alto (CAHA, Almería, Spain). CARMENES is funded by the German Max-Planck-Gesellschaft (MPG), the Spanish Consejo Superior de Investigaciones Científicas (CSIC), the European Union through FEDER/ERF FICTS-2011-02 funds, and the members of the CARMENES Consortium (Max-Planck-Institut für Astronomie, Instituto de Astrofísica de Andalucía, Landessternwarte Königstuhl, Institut de Ciències de l'Espai, Insitut für Astrophysik Göttingen, Universidad Complutense de Madrid, Thüringer Landessternwarte Tautenburg, Instituto de Astrofísica de Canarias, Hamburger Sternwarte, Centro de Astrobiología and Centro Astronómico Hispano-Alemán), with additional contributions by the Spanish Ministry of Economy, the German Science Foundation through the Major Research Instrumentation Programme and DFG Research Unit FOR2544 "Blue Planets around Red Stars", the Klaus Tschira Stiftung, the states of Baden-Württemberg and Niedersachsen, and by the Junta de Andalucía. This article is based on observations made in the Observatorios de Canarias del IAC with the TNG telescope operated on the island of La Palma by the Galileo Galilei Fundation, in the Observatorio del Roque de los Muchachos (ORM). HARPS-N data were taken under observing programs CAT17A-91, A36TAC-12 and OPT17B-59. This work is partly financed by the Spanish MINECO through grants ESP2016-80435-C2-1-R, ESP2016-80435-C2-2-R and AYA2016-79425-C3-3-P. This work was also supported by JSPS KAKENHI Grants Numbers JP16K17660 and JP18H01265.

\section{References}

Auvergne, M., Bodin, P., Boisnard, L., et al. 2009, A\&A, 506, 411 Barragán, O., Grziwa, S., Gandolfi, D., et al. 2016, AJ, 152, 193 Barragán, O., Gandolfi, D., Dai, F., et al. 2018, A\&A, 612, A95 Borucki, W. J., Koch, D., Basri, G., et al. 2010, Science, 327, 977 Bruntt, H., Bedding, T. R., Quirion, P.-O., et al. 2010, MNRAS, 405, 1907

Cleveland, W. S. 1979, ASA, 74, 829

Cosentino, R., Lovis, C., Pepe, F., et al. 2012, in Ground-based and Airborne Instrumentation for Astronomy IV, Proc. SPIE, 8446, 84461 V da Silva, L., Girardi, L., Pasquini, L., et al. 2006, A\&A, 458, 609 Deming, D., Knutson, H., Kammer, J., et al. 2015, ApJ, 805, 132

Doyle, A. P., Davies, G. R., Smalley, B., Chaplin, W. J., \& Elsworth, Y. 2014, MNRAS, 444, 3592

Dumusque, X., Boisse, I., \& Santos, N. C. 2014, ApJ, 796, 132

Foreman-Mackey, D., Hogg, D. W., Lang, D., \& Goodman, J. 2013, PASP, 125, 306

Fulton, B. J., \& Petigura, E. A. 2018, AJ, 156, 264

Fulton, B. J., Petigura, E. A., Howard, A. W., et al. 2017, AJ, 154, 109

Gustafsson, B., Edvardsson, B., Eriksson, K., et al. 2008, A\&A, 486, 951

Hirano, T., Fukui, A., Mann, A. W., et al. 2016, ApJ, 820, 41

Hirano, T., Dai, F., Gandolfi, D., et al. 2018, AJ, 155, 127

Kipping, D. M. 2013, MNRAS, 435, 2152

Kobayashi, N., Tokunaga, A. T., Terada, H., et al. 2000, in Optical and IR Telescope Instrumentation and Detectors, eds. M. Iye \& A. F. Moorwood, Proc. SPIE, 4008, 1056

Kovács, G., Zucker, S., \& Mazeh, T. 2002, A\&A, 391, 369

Kreidberg, L. 2015, PASP, 127, 1161

Lindegren, L., Hernández, J., Bombrun, A., et al. 2018, A\&A, 616, A2

Luger, R., Kruse, E., Foreman-Mackey, D., Agol, E., \& Saunders, N. 2018, AJ, 156, 99

Luque, R., Nowak, G., Pallé, E., et al. 2018, A\&A, 620, A171

Luri, X., Brown, A. G. A., Sarro, L. M., et al. 2018, A\&A, 616, A9

Mandel, K., \& Agol, E. 2002, ApJ, 580, L171

Niraula, P., Redfield, S., Dai, F., et al. 2017, AJ, 154, 266

Oscoz, A., Rebolo, R., López, R., et al. 2008, in Ground-based and Airborne Instrumentation for Astronomy II, Proc. SPIE, 7014, 701447

Pepe, F., Cameron, A. C., Latham, D. W., et al. 2013, Nature, 503, 377

Piskunov, N., \& Valenti, J. A. 2017, A\&A, 597, A16

Poznanski, D., Prochaska, J. X., \& Bloom, J. S. 2012, MNRAS, 426, 1465

Prieto-Arranz, J., Palle, E., Gandolfi, D., et al. 2018, A\&A, 618, A116

Quirrenbach, A., Amado, P. J., Caballero, J. A., et al. 2014, in Ground-based and Airborne Instrumentation for Astronomy V, Proc. SPIE, 9147, 91471F 
Reiners, A., Ribas, I., Zechmeister, M., et al. 2018, A\&A, 609, L5

Scott, N. J., Howell, S. B., \& Horch, E. P. 2016, in Optical and Infrared Interferometry and Imaging V, Proc. SPIE, 9907, 99072R

Scott, N. J., Howell, S. B., Horch, E. P., \& Everett, M. E. 2018, PASP, 130 054502

Southworth, J. 2011, MNRAS, 417, 2166

Torres, G., Andersen, J., \& Giménez, A. 2010, A\&ARv, 18, 67

Trifonov, T., Kürster, M., Zechmeister, M., et al. 2018, A\&A, 609, A117

Van Eylen, V., \& Albrecht, S. 2015, ApJ, 808, 126

Van Eylen, V., Agentoft, C., Lundkvist, M. S., et al. 2018, MNRAS, 479, 4786 van Leeuwen F. 2007, A\&A, 474, 653

Winn, J. N. 2010, Exoplanet Transits and Occultations (Tucson, AZ: University of Arizona Press)

Winn, J. N., Petigura, E. A., Morton, T. D., et al. 2017, AJ, 154, 270

Zechmeister, M., \& Kürster, M. 2009, A\&A, 496, 577

Zechmeister, M., Reiners, A., Amado, P. J., et al. 2018, A\&A, 609, A12

Zeng, L., Sasselov, D. D., \& Jacobsen, S. B. 2016, ApJ, 819, 127

${ }^{1}$ Instituto de Astrofísica de Canarias (IAC), 38205 La Laguna, Tenerife, Spain

e-mail: epalle@iac.es

2 Departamento de Astrofísica, Universidad de La Laguna (ULL), 38206 La Laguna, Tenerife, Spain

3 Dipartimento di Fisica, Università di Torino, Via P. Giuria 1, 10125 Torino, Italy

${ }^{4}$ Department of Earth and Planetary Sciences, Tokyo Institute of Technology, 2-12-1 Ookayama, Meguro-ku, Tokyo 152-8551, Japan

${ }^{5}$ Leiden Observatory, Leiden University, 2333CA Leiden, The Netherlands

6 Department of Space, Earth and Environment, Chalmers University of Technology, Onsala Space Observatory, 43992 Onsala, Sweden

7 Department of Astronomy, The University of Tokyo, 7-3-1 Hongo, Bunkyo-ku, Tokyo 113-0033, Japan

${ }^{8}$ Department of Astrophysical Sciences, Princeton University, 4 Ivy Lane, Princeton, NJ 08544, USA

9 Department of Physics and Kavli Institute for Astrophysics and Space Research, Massachusetts Institute of Technology, Cambridge, MA 02139, USA
${ }^{10}$ Institut de Ciències de l' Espai (ICE, CSIC), C/Can Magrans, s/n, Campus UAB, 08193 Bellaterra, Spain

${ }^{11}$ Institut d'Estudis Espacials de Catalunya (IEEC), 08034 Barcelona, Spain

12 Stellar Astrophysics Centre, Department of Physics and Astronomy, Aarhus University, Ny Munkegade 120, 8000 Aarhus C, Denmark

13 Instituto de Astrofísica de Andalucía (IAA-CSIC), Glorieta de la Astronomía s/n, 18008 Granada, Spain

${ }^{14}$ Centro de Astrobiología (CSIC-INTA), ESAC Campus, Camino Bajo del Castillo s/n, 28692 Villanueva de la Cañada, Madrid, Spain

15 Institute of Planetary Research, German Aerospace Center, Rutherfordstrasse 2, 12489 Berlin, Germany

${ }^{16}$ Institute of Geological Sciences, Freie Universität Berlin, Malteserstr. 74-100, 12249 Berlin, Germany

${ }^{17}$ Center for Astronomy and Astrophysics, Technische Universität Berlin, Hardenbergstr. 36, 10623 Berlin, Germany

18 Department of Astronomy and McDonald Observatory, University of Texas at Austin, 2515 Speedway, Stop C1400, Austin, TX 78712, USA

19 Okayama Astrophysical Observatory, National Astronomical Observatory of Japan, NINS, Asakuchi, Okayama 719-0232, Japan

20 Thüringer Landessternwarte Tautenburg, Sternwarte 5, 07778 Tautenburg, Germany

${ }^{21}$ Rheinisches Institut für Umweltforschung an der Universität zu Köln, Aachener Strasse 209, 50931 Köln, Germany

22 Max-Planck-Institut für Astronomie, Königstuhl 17, 69117 Heidelberg, Germany

23 Astrobiology Center, NINS, 2-21-1 Osawa, Mitaka, Tokyo 1818588, Japan

${ }^{24}$ National Astronomical Observatory of Japan, NINS, 2-21-1 Osawa, Mitaka, Tokyo 181-8588, Japan

25 Landessternwarte, Zentrum für Astronomie der Universtät Heidelberg, Königstuhl 12, 69117 Heidelberg, Germany

26 Astronomy Department and Van Vleck Observatory, Wesleyan University, Middletown, CT 06459, USA

${ }^{27}$ Institut für Astrophysik, Georg-August-Universität, Friedrich-HundPlatz 1, 37077 Göttingen, Germany 


\section{Appendix A: Additional table}

Table A.1. Summary of the system parameters of K2-285 determined in Sect. 2 using only the fit to the photometric data from the K2 mission, and in Sect. 5 with the Pyaneti code fitting the photometric and RV data simultaneously.

\begin{tabular}{|c|c|c|c|c|c|}
\hline Parameter & $\mathrm{K} 2-285 \mathrm{~b}$ & $\mathrm{~K} 2-285 \mathrm{c}$ & $\mathrm{K} 2-285 \mathrm{~d}$ & $\mathrm{~K} 2-285 \mathrm{e}$ & Stellar signal \\
\hline \multicolumn{6}{|l|}{ Model fits to K2 data only } \\
\hline Orbit inclination $i_{\mathrm{p}}\left({ }^{\circ}\right)$ & $87.0_{-2.0}^{+2.0}$ & $88.0_{-1.0}^{+1.0}$ & $89.2_{-0.9}^{+0.6}$ & $89.4_{-0.6}^{+0.4}$ & \\
\hline Semi-major axis $a\left(R_{*}\right)$ & $11.0_{-3.0}^{+2.0}$ & $17.0_{-4.0}^{+4.0}$ & $30.0_{-6.0}^{+3.0}$ & $45.0_{-10.0}^{+5.0}$ & \\
\hline Transit epoch $T_{0}$ (JD-2 454833 ) & $2910.3753_{-0.0007}^{+0.0006}$ & $2911.5384_{-0.0007}^{+0.0004}$ & $2912.201_{-0.001}^{+0.001}$ & $2908.897_{-0.002}^{+0.003}$ & \\
\hline Planet radius $R_{\mathrm{p}}\left(R_{\oplus}\right)$ & $2.62_{-0.04}^{+0.05}$ & $3.7_{-0.3}^{+0.3}$ & $2.57_{-0.06}^{+0.09}$ & $2.01_{-0.09}^{+0.20}$ & \\
\hline Orbital period $P_{\text {orb }}$ (days) & $3.47175_{-0.00005}^{0.00004}$ & $7.13804_{-0.00010}^{+0.00007}$ & $10.4560_{-0.0003}^{0.0004}$ & $14.7634_{-0.0006}^{0.0007}$ & \\
\hline Impact parameter $b$ & $0.5_{-1.0}^{+0.8}$ & $0.5_{-0.7}^{+0.7}$ & $0.4_{-0.4}^{+0.3}$ & $0.5_{-0.3}^{+0.2}$ & \\
\hline Transit depth & $0.00097_{-0.00003}^{+0.00004}$ & $0.00210_{-0.0002}^{+0.0003}$ & $0.00100_{-0.00004}^{+0.00007}$ & $0.00068_{-0.00006}^{+0.00009}$ & \\
\hline Transit duration $\tau_{14}(\mathrm{~h})$ & $2.57 \pm 0.02$ & $3.08 \pm 0.03$ & $2.96 \pm 0.05$ & $2.76 \pm 0.07$ & \\
\hline Linear limb-darkening coefficient $u_{1}$ & $0.2_{-0.1}^{+0.2}$ & $0.5_{-0.2}^{+0.3}$ & $0.3_{-0.3}^{+0.4}$ & $0.9_{-0.5}^{+0.5}$ & \\
\hline Quadratic limb-darkening coefficient $u_{2}$ & $0.06_{-0.05}^{+0.07}$ & $0.38_{-0.09}^{+0.10}$ & $0.18_{-0.09}^{+0.30}$ & $0.0_{-0.2}^{+0.3}$ & \\
\hline Eccentricity $^{a} e$ & $0^{-0.05}$ & & & & \\
\hline Longitude of periastron ${ }^{a} \omega_{\star}\left(^{\circ}\right)$ & 90 & & & & \\
\hline
\end{tabular}

\section{Model parameters: Pyaneti}

Orbital period $P_{\text {orb }}$ (days)

Transit epoch $T_{0}$ (JD-2450000)

Scaled planet radius $R_{\mathrm{p}} / R_{\star}$

Impact parameter $b$

$\sqrt{e} \sin \omega_{\star}^{a}$

$\sqrt{e} \cos \omega_{\star}^{a}$

Doppler semi-amplitude $K\left(\mathrm{~m} \mathrm{~s}^{-1}\right)$

Systemic velocity $\gamma_{\text {CARMENES }}\left(\mathrm{km} \mathrm{s}^{-1}\right)$

Systemic velocity $\gamma_{\text {HARPS-N }}\left(\mathrm{km} \mathrm{s}^{-1}\right)$

Limb-darkening coefficient $q_{1}^{b}$

Limb-darkening coefficient $q_{2}^{b}$

\section{Derived parameters: Pyaneti}

Planet mass $M_{\mathrm{p}}\left(M_{\oplus}\right)$

Planet radius $R_{\mathrm{p}}\left(R_{\oplus}\right)$

Planet density $\rho_{\mathrm{p}}\left(\mathrm{g} \mathrm{cm}^{-3}\right)$

Surface gravity $g_{\mathrm{p}}\left(\mathrm{cm} \mathrm{s}^{-2}\right)$

Surface gravity ${ }^{c} g_{\mathrm{p}}\left(\mathrm{cm} \mathrm{s}^{-2}\right)$

Scaled semi-major axis $a / R_{\star}$

Semi-major axis $a(\mathrm{AU})$

Orbit inclination $i_{\mathrm{p}}\left(^{\circ}\right)$

Transit duration $\tau_{14}(\mathrm{~h})$

Equilibrium temperature ${ }^{d} T_{\text {eq }}(\mathrm{K})$

Insolation $F\left(F_{\oplus}\right)$

Stellar density (from light curve)

Linear limb-darkening coefficient $u_{1}$

Quadratic limb-darkening coefficient $u_{2}$

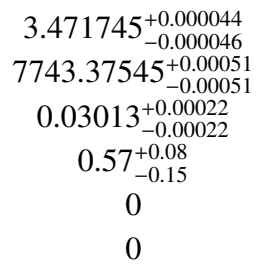

$7.138048_{-0.000063}^{+0.000072}$

$7.10 .45582_{-0.00023}^{+0.00025}$

$7744.53906_{-0.00037}^{+0.00039}$

$0.0411476_{-0.003004}^{+0.000304}$

$4.62_{-0.65}^{+0.58}$

$0.00130_{-0.00068}^{+0.00071}$

$0.00168_{-0.00076}^{+0.000081}$

$0.272_{-0.098}^{+0.092}$

$0.62^{+0.22}$
$0.05_{-0.04}^{+0.04}$

0

0

$10.45582_{-0.00023}^{+0.00025}$
$7745.20100_{-0.000073}^{+0.00076}$
$0.0288961_{-0.0002822}^{+0.000320}$
$0.20_{-0.05}^{+0.04}$
0
0

$5.90_{-0.80}^{+0.86}$
$14.76289_{-0.00061}^{+0.00065}$

$7741.8969^{+0.0020}$

$0.0227044^{+0.0003527}$

$0.17_{-0.07}^{+0.04}$

0

0

$1.69_{-0.72}^{+0.74}$
$12.102_{-0.056}^{+0.067}$ $7985.84_{-0.30}^{+0.30}$

$29_{-0.81}^{+0.80}$

$\begin{array}{cccc} & & & \\ 9.68_{-1.37}^{+1.21} & 15.68_{-2.13}^{+2.28} & <6.5 & <10.7 \\ 2.59_{-0.06}^{+0.06} & 3.53_{-0.08}^{+0.08} & 2.48_{-0.06}^{+0.06} & 1.95_{-0.05}^{+0.05} \\ 3.07_{-0.45}^{+0.45} & 1.95_{-0.28}^{+0.32} & & \\ 1420.1_{-202.7}^{+191.8} & 1234.1_{-172.4}^{+191.7} & & \\ 1165.9_{-169.9}^{+141.6} & 1799.3_{-242.3}^{+260.7} & & \\ 10.43_{-0.14}^{+0.14} & 22.51_{-0.20}^{+0.20} & 32.20_{-0.45}^{+0.45} & 49.43_{-0.73}^{+0.41} \\ 0.03817_{-0.00095}^{+0.00095} & 0.0824_{-0.0018}^{+0.0018} & 0.1178_{-0.0029}^{+0.0029} & 0.18041_{-0.0043}^{+0.0042} \\ 86.846_{-0.041}^{+0.041} & 89.8610_{-0.0012}^{+0.0012} & 89.6431_{-0.0051}^{+0.0049} & 89.7994_{-0.0030}^{+0.0016} \\ 2.180_{-0.028}^{+0.029} & 2.520_{-0.021}^{+0.022} & 2.504_{-0.033}^{+0.035} & 2.300_{-0.019}^{+0.034} \\ 1088.9_{-21.9}^{+22.1} & 741.4_{-14.5}^{+14.5} & 619.9_{-12.5}^{+12.7} & 500.9_{-9.9}^{+10.1} \\ 234.31_{-18.28}^{+19.58} & 50.35_{-3.83}^{+4.05} & 24.61_{-1.93}^{+2.07} & 10.50_{-0.81}^{+0.87} \\ 1.782_{-0.069}^{+0.071} & & & \\ 0.646_{-0.097}^{+0.081} & & & \\ -0.129_{-0.158}^{+0.170} & & & \end{array}$

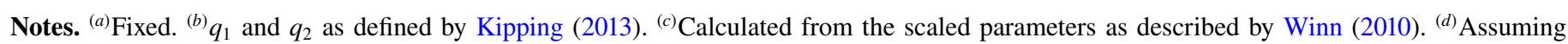
albedo $=0$. 